\title{
Generation and validation of an anti-human PANK3 mouse monoclonal antibody
}

Sunada Khadka ${ }^{1,2,3}$, Long Vien ${ }^{4}$, Paul Leonard ${ }^{5}$, Laura Bover ${ }^{4,6}$ and Florian Muller ${ }^{5}$

1. Department of Cancer Systems Imaging, The University of Texas MD Anderson Cancer Center, Houston, TX, USA

2. Department of Cancer Biology, The University of Texas MD Anderson Cancer Center, Houston, TX, USA

3. MD Anderson UT Health Graduate School of Biomedical Sciences, Houston, TX, USA

4. Department of Immunology, The University of Texas MD Anderson Cancer Center, Houston, TX, USA

5. Institute of Applied Cancer Science, The University of Texas MD Anderson Cancer Center, Houston, TX, USA

6. Department of Genomics Medicine, The University of Texas MD Anderson Cancer Center, Houston, TX, USA

7. Sporos Bioventures, Houston, TX, USA

Corresponding authors: sunadaxkhadka@gmail.com; aettius@aol.com

\section{Keywords}

Pantothenate Kinases, PANK3, monoclonal antibody

Abstract:

Coenzyme $A(C \circ A)$ is an essential co-factor at the intersection of diverse metabolic pathways. Cellular CoA biosynthesis is regulated at the first committed stepphosphorylation of pantothenic acid—catalyzed by pantothenate kinases (PANK1,2,3 in humans, PANK3 being the most highly expressed). Despite the critical importance of CoA 
24 in metabolism, the differential roles of PANK isoforms remain poorly understood. Our

25 investigations of PANK proteins as precision oncology collateral lethality targets

26 (PANK1 is co-deleted as part of the PTEN locus in some highly aggressive cancers) were

27 severely hindered by a dearth of commercial antibodies that can reliably detect endogenous PANK3. While we successfully validated commercial antibodies for PANK1 and PANK2 using CRISPR knockout cell lines, we found no commercial antibody that could detect endogenous PANK3. We therefore set out to generate a mouse monoclonal antibody against human PANK3 protein. We demonstrate that clone (Clone MDA-29962A) can reliably detect endogenous PANK3 protein in cancer cell lines, with bandspecificity confirmed by CRISPR PANK3 knockout cell lines. Sub-cellular fractionation indicates that PANK3 is overwhelmingly cytosolic and expressed broadly across cancer cell lines. PANK3 monoclonal antibody MDA-299-62A should prove a valuable tool for researchers investigating this understudied family of metabolic enzymes in health and disease.

\section{Introduction:}

Coenzyme $A(C o A)$ is a central co-factor for more than $4 \%$ of known cellular enzymes and is involved in hundreds of biochemical reactions in the mammalian cells metabolic pathways such as synthesis and oxidation of fatty acids, oxidative anaplerosis of pyruvate in the TCA cycle and the synthesis of lipids, isoprenoids and sterols, amino

44 acid metabolism, and porphyrin synthesis $(1,2)$. Additionally, CoA derived 45 phosphopantetheine prosthetic group is also critical for enzymes involved in fatty acid, 
47 first and the committed step of de novo CoA biosynthesis (5). Being the rate-limiting enzymes of the pathway, PANKs phosphorylate pantothenic acid (vitamin B5), which is converted to CoA in a series of four evolutionarily conserved enzymatic reactions $(1,5)$. The family of PANKs constitutes four catalytically active isoforms-nuclear PANK1a and cytosolic PANK1 $\beta$ (both encoded by PANK1), mitochondrial (intermembrane space) PANK2 and cytosolic PANK3 (6). PANK isoforms are also differentially expressed and regulated, enabling these proteins to sense and maintain the levels of CoA and its thioesters differentially in specific cellular compartment $(2,7)$. PANK proteins have been studied extensively in the context of normal mammalian physiology and pathologies that arise due to their dysregulation (8-12). Inactivating mutations in PANK2 protein have been

57 linked to a neurodegenerative condition called pantothenate kinase-associated neurodegeneration (PKAN) in humans (13-17). Similarly, cancers with homozygous (biallelic) deletion of the tumor suppressor PTEN, that are typically highly aggressive, poor prognosis and refractory to treatment (18-20), can show co-deletion of the neighboring gene PANK1, making the redundant PANK proteins attractive therapeutic targets in these cancers $(21,22)$. Despite the obvious importance of PANKs in CoA biosynthesis, the lack of reliable antibodies that can detect and distinguish endogenous PANK isoform proteins, has significantly thwarted efforts to better understand their role in both normal physiology and in pathological contexts, as well as characterize them as therapeutic targets. Generation of antibodies against PANK proteins, especially PANK3, has been

67 significantly challenging given the extreme homology between the typical host species isozymes pose additional challenges in generating isozyme-specific antibodies. While 
many commercial antibodies may detect unphysiological levels of overexpressed PANK proteins, few antibodies are able to detect endogenously expressed PANK. Through an extensive and laborious screening of commercially available antibodies, we validated the antibodies for PANK1 and PANK2 for the detection of endogenous proteins using PANKspecific CRISPR KO cell lines. However, we could not find commercial antibodies capable of detecting endogenous human PANK3 protein.

Here, we describe the generation, optimization and validation of a mouse monoclonal antibody (mAb) (MDA-299-62A) against human PANK3 protein. We demonstrate that MDA-299-62A can reliably detect purified recombinant human PANK3, and endogenous PANK3 protein in cancer cell lysates with band specificity validated by PANK3 CRISPR knockout cancer cell lines. The PANK3 antibody generated in this study could serve as a reliable tool for quantifying endogenous PANK3 protein and should prove to be valuable for studies investigating these enzymes in health and disease.

\section{Methods:}

\section{Protein Expression and Purification}

The pET28a plasmid vector containing DNA sequences encoding the PANK3 protein (residues pro12 to Asn368) was purchased from Addgene (25518) and transformed into both E. coli BL21 (DE3) and E. coli Rosetta2 (DE3) strains. The E. coli cells were grown in Terrific Broth media at $37^{\circ} \mathrm{C}$ until the optical density at $600 \mathrm{~nm}$ reached 0.5 absorbance units. The temperature of the culture was then reduced to $18^{\circ} \mathrm{C}$ prior to induction of the recombinant protein expression by the addition of $1 \mathrm{mM}$ isopropyl B-D-1-thiogalactopyranoside (IPTG). All protein purification steps were performed at $4^{\circ} \mathrm{C}$ 
92 After harvesting the cells by centrifugation, the $E$. coli cells were lysed by re-suspending

93 the pellet in $10 \mathrm{mM}$ Tris $\mathrm{pH} 7.5,0.5 \mathrm{M} \mathrm{NaCl}, 5 \%$ glycerol, $5 \mathrm{mM}$ Imidazole, complete

94 protease inhibitors (Roche), $30 \mu \mathrm{g} \mathrm{mL}{ }^{-1}$ DNase and $500 \mu \mathrm{g} \mathrm{mL}^{-1}$ lysozyme at $\mathrm{pH} 7.5$ and

95 lysed by sonication. The lysate was centrifuged at 20,000 rpm for 45 min to remove insoluble material. The recombinant protein was purified from the clarified lysate using a

$972 \mathrm{~mL}$ Ni-NTA column pre-equilibrated in $10 \mathrm{mM}$ Tris $\mathrm{pH} 7.5,0.5 \mathrm{M} \mathrm{NaCl}, 5 \%$ glycerol, 5 mM Imidazole buffer. The Ni-NTA column was washed with $10 \mathrm{mM}$ Tris- $\mathrm{HCl}, 0.5 \mathrm{M} \mathrm{NaCl}$, $5 \%$ glycerol and $30 \mathrm{mM}$ imidazole at $\mathrm{pH}$ 7.5. The bound proteins were eluted at $10 \mathrm{mM}$ Tris- $\mathrm{HCl}, 0.5 \mathrm{M} \mathrm{NaCl}, 5 \%$ glycerol, $250 \mathrm{mM}$ Imidazole at $\mathrm{pH}$ 7.5. The fractions were protein eluted were assessed by SDS PAGE.

\section{Monoclonal antibody production and purification}

All animal experiments were performed using protocols approved by the Institutional Animal Care and Use Committee (IACUC). Antibody production using hybridoma technology and purifications were done at the MD Anderson Monoclonal Antibody Core. A detailed protocol on the protocol has been described. (23). Briefly,

107 purified recombinant PANK3 protein was emulsified using the incomplete Freund 108 Adjuvant (IFA, In-vivoGen) in a 1:1 ratio. Two NZBWF1/J mice and one BALB/c mice were used for immunization. $10 \mu \mathrm{g}$ of recombinant protein (20 $\mu$ l volume total) was administered through the foot-pad route every two days for the first five injections, and 111 the dose was raised to $15 \mu \mathrm{g}$ for three booster injections administered weekly. After 112 completion of immunization, on day 31, mice were sacrificed and the popliteal lymph 113 nodes and spleen from the immunized mice with the highest serum titer were harvested 114 using sterile methods. B cell isolated either from the lymph node or the spleen were fused 
115 with Sp20 murine myeloma cells, and selected in hypoxanthine-aminopterin-thymidine

116 (HAT) medium, and single cell clones were identified. Enzyme linked immunoassay

117 (ELISA) screening was performed using the supernatant from each positive hybridoma

118 clone to determine the efficiency of target protein binding by the mAb containing

119 supernatants. Western blot was performed on HeLa cells to identify the clones that could

120 detect endogenous PANK3 protein. The selected hybridoma clones were expanded and

$121300 \mathrm{~mL}$ of $\mathrm{mAb}$ rich supernatants were collected and filtered using a $0.45 \mu \mathrm{m}$ membrane.

122 The supernatants were added to Protein A columns for affinity chromatography

123 purification. The antibodies captured in the column were eluted using elution buffer $(0.1$

$124 \mathrm{M}$ glycine- $\mathrm{HCl}, \mathrm{pH}=3$ ) that was neutralized with $1 \mathrm{M}$ Tris- $\mathrm{HCl}$ buffer. Overnight dialysis was

125 performed in PBS to concentrate and preserve the antibody activity in a neutral buffer.

126

128

129

130

131

132

133

134

135

136

137

\section{ELISA Assay}

ELISA plates were first coated with $100 \mu$ of coating buffer containing the recombinant target protein used for immunization at $1 \mu \mathrm{g} / \mathrm{ml}$ and incubated at $4^{\circ} \mathrm{C}$ overnight. After 3X PBST wash, the plate was blocked with $100 \mu$ of ELISA blocking buffer at RT for 1 hour. $50 \mu$ of diluted serum from the immunized mice or the media supernatant from each hybridoma clone, which serve as the primary antibody, were added to individual wells respectively in the corresponding screenings, and incubated for 1 hour. The plate was then washed $3 \mathrm{X}$ in PBST, and $50 \mu \mathrm{l}$ of goat anti-mouse HRP conjugated secondary antibody was added to each well and incubated for one hour. After 4X PBST washes, horseradish peroxidase (HRP) substrate TMB was added and the reaction was stopped by addition of $\mathrm{H}_{2} \mathrm{SO} 4$ after 30 mins. The OD $450 \mathrm{~nm}$ values were then measured using a spectrophotometer. 
140 The cell lines used in this work were HeLa (CVCL_0030, Cervical Carcinoma), 537 MEL

141 (CVCL_8052, Melanoma), HAP1(CVCL_YO91), SKMEL-5 (CVCL_0527, Melanoma), 142 SKMEL28 (CVCL_0526, Melanoma), SKMEL2 (CVCL_0069, Melanoma), G59 143 (CVCL_N729, Glioblastoma), D423-MG (CVCL_1160, Glioblastoma), referred to as D423 144 in the figures, LN319, a sub-clone of LN-99267, (CVCL_3958, Glioblastoma), D502 145 (CVCL_1162, Glioblastoma) and HEK293T (CVCL_0063). The cell lines were 146 authenticated at the MD Anderson Cytogenetics and Cell Authentication Core. The cell 147 lines were obtained from the following sources: HeLa (ATCC), 537 MEL (NCI), HAP1 148 (Horizon Discovery), SKMEL-5 (ATCC), SKMEL-2 (ATCC), SKMEL-28 (ATCC), G59 149 (Prof.Dr. Katrin Lamszus, Universitätsklinikum Hamburg-Eppendorf), D423 (Darrel 150 Bigner, Duke University), LN319 (ATCC), D502 (ATCC), HEK 293T(ATCC). All cells were 151 maintained in RPMI medium with 2mM glutamine (Cellgro/Corning \#10-040-CV) 152 supplemented with 10\%FBS (Gibco/Life Technologies \#16140-071) and 1\% pen-strep 153 (Gibco/Life Technologies\#15140-122).

Generation of CRISPR KO Clones CRISPR KO was performed using the Santa Cruz dual plasmid CRISPR system. PANK CRISPR plasmids (PANK1 (sc-408890), PANK2 (sc-405120) and PANK3 sc157 409325) and PANK HDR plasmids (PANK1 (sc-408890-HDR), PANK2 (sc-405120-HDR) 158 and PANK3 (sc-409325-HDR) plasmids were purchased from Santa Cruz 159 Biotechnologies. $1 \mu \mathrm{g}$ of PANK plasmids and $1 \mu \mathrm{g}$ of PANK HDR plasmids were mixed 
160 together in $140 \mu$ plasmid transfection medium (sc-108062). In a separate tube, $5 \mu$ of

161 UltraCruz® (sc-395739) transfection reagent was added to $145 \mu$ l transfection medium,

162 and the plasmid and transfection reagents were mixed together and incubated for 10

163 minutes at RT. The cell culture medium in the target cells was replaced with fresh medium

164 and the CRISPR/HDR plasmid mix were slowly added to the plates, and the plates were

165 gently shaken to allow for a homogenous mixing. 24 hours later the medium was replaced

166 with fresh medium and the cells were subjected to puromycin selection for a week. The

167 cells were then trypsinized, washed in PBS and resuspended in FACS buffer

168 (1\%FBS+0.5mM EDTA in PBS). Single cell sorting was performed with RFP channel as

169 a gate using a BD FACS ARIA Cells sorter. Confirmation of knockout was done with

170 western blots.

171 Generation of stable dox inducible PANK3 shRNA cell lines

172

Third generation lentivirus packaging system was used to generate the stable dox

173 inducible shRNA expressing cells. Recombinant lentiviral particles were generated by

174 transient transfection of HEK 293T cells following a standard protocol. ShRNA plasmids

175 encoding a doxycycline inducible shRNA, hygromycin resistance marker and red

176 fluorescent protein (RFP) were purchased from Cellecta. For transfection, $10 \mu \mathrm{g}$ of the

177 shRNA plasmid, were mixed with $5 \mu \mathrm{g}$ of pMd2g, and $5 \mu \mathrm{g}$ of pREV and $5 \mu \mathrm{g}$ of p8.74

178 and $10 \mu \mathrm{g}$ of shRNA vector and the plasmids were transfected into HEK 293T cells plated

179 in $100 \mathrm{~mm}^{2}$ dishes. Fresh medium was replaced after 12 hours. Viral supernatants were

180 collected, and fresh medium added on the HEK 293T cells every 24 hours until 48 hours

181 since first media change. Cell debris were eliminated by centrifugation at $500 \mathrm{~g}$, and the

182 supernatant containing the virus particles was sterile filtered through $0.45 \mu \mathrm{m}$ filter. To 
183 infect the target cells, the filtered viral particles were mixed with an equal volume of fresh

184 medium and polybrene $(8 \mu \mathrm{g} / \mathrm{ml})$ and added to target cells. The media on target cells

185 were replaced with fresh media 24 hours after infection. After 48 hours since infection, $186200 \mu \mathrm{g} / \mathrm{ml}$ hygromycin was added for selection. Knockdown of PANK3 was induced by

187 addition of $2 \mu \mathrm{g} / \mathrm{ml}$ dox for $48-72$ hours and knockdown was confirmed by western blot.

\section{Western Blot}

\section{Whole cell lysate preparation}

190 Cells were grown in 6 well plates for $48-72$ hours and the lysates were harvested by 191 washing the cells twice with ice cold phosphate-buffered saline (PBS). Ice-cold RIPA 192 buffer with protease (cOmplete ${ }^{\mathrm{TM}}$ mini, Roche\#11836153001) and phosphatase inhibitors 193 (PhosSTOP, Roche, \#5892970001), were added and the samples were then sonicated. fractionation kit (\#78840), using the manufacturer's directions. Briefly, cells were 197 trypsinized and the pellets were washed with ice-cold PBS. Ice cold cytoplasmic 198 extraction buffer with 1X protease inhibitor cocktail was added to re-suspend the pellet 199 and the sample was incubated on ice for 10 minutes with gentle mixing. The cell lysate was then centrifuged at $500 \mathrm{~g}$ for 5 minutes at $4^{\circ} \mathrm{C}$, and the cytosolic fraction in the

201 supernatant was transferred to a pre-chilled tube, without agitating the pellet. Ice-cold membrane extraction buffer was added to the pellet, and the tube was vortexed at the 
204 tube was then centrifuged at $3000 \mathrm{~g}$ for 5 minutes, and membrane fraction was isolated 205 from the supernatant.

206 BCA assay (ThermoFisher, \#23227), was used to determine the protein concentration.

207 Proteins were separated by Nu-PAGE SDS-PAGE (4-12\% gradient) and transferred onto 208 nitrocellulose membranes using the semi-dry method (TransBlot turbo). Effective transfer

209 of proteins on the membrane was verified with Ponceau S staining. 5\% non-fat dry milk 210 in tris-buffered saline (TBS) with $0.1 \%$ Tween 20 (TBST) was used as the blocking agent 211 to block the non-specific sites on membrane. Primary antibodies (PANK1 CST 1:1000; 212 PANK2 Origene 1:1000; PANK3 5 $\mathrm{g} / \mathrm{ml}$, TPI1 ProteinTech; 10713-1-AP 1:10000,) were 213 added to the membranes and incubated overnight at $4^{\circ} \mathrm{C}$ with gentle rocking. On the 214 second day, the membranes were washed $3 x$ for 5 min with TBST. The membranes were 215 then incubated in HRP tagged secondary antibody (1:5000) for 1 hour with gentle rocking 216 and then washed $3 x$ for 5 min with TBST. Then the membranes were incubated first with

217 ECL substrate (32106) and X-ray films were exposed in a dark room and developed with 218 different exposure times. If the bands were not visible, ThermoScientific SuperSignal 219 West Femto (34096) was used as a substrate and the membranes developed on the X220 ray films.

221 Results

1. CRISPR-Validated commercial antibodies for PANK1 and PANK2, but not PANK3

To identify commercially available antibodies that can detect endogenous PANK 225 proteins, we tested antibodies from diverse commercial suppliers. We found many 226 commercial antibodies that can detect highly overexpressed unphysiological levels of 
227 PANKs, such as those obtained by transient transfection of PANK overexpressing plasmids. But very few can specifically detect endogenous expression of PANK. Additionally, the band specificities of these antibodies were not validated with a proper negative control by using a PANK deficient or PANK CRISPR knockout cell lines. To mitigate these issues, we purchased CRISPR knockout clones of PANK1, PANK2 and PANK3 in HAP1 cells (Figure 1A) from Horizon Discovery. HAP1 is a near-haploid cell line derived from KBM-7, a chronic myelogenous leukemia cell line. Due to its haploidy, HAP1 is an ideal model for genetic manipulations with CRISPR, and holds a great potential for genetic screening studies (24). Since HAP1 cells have almost one copy of most genes, the CRISPR induced heterozygous mutation artifacts (as in a diploid cell) are eliminated, allowing for easy and efficient generation of knockout clones. PANK isoform KOs in HAP1 cells were initially custom-made to order, but they are now available off-the shelf for other investigators to use. Additionally, we also independently generated knockout clones of PANK1, PANK2 and PANK3 in multiple different cancer cell lines to validate the band specificity and serve as a negative control for the antibodies

(Figure 1C-E). We found that CST\#23887S rabbit monoclonal antibody against PANK1 detects PANK1 protein at the expected size of $50 \mathrm{kD}$ which disappears in two independent

244 CRISPR knockout clones in HAP1 and HeLa cells (Figure 1A and C). Further validation of CST PANK1 antibody was also done in PANK1 endogenous genomic deleted cell lines 246 like 537 MEL and G59 and other PANK1 intact cell lines, which further reinforced the 247 band specificity of the antibody (Figure 1B). Similarly, we found that Origene's \#TA501321 mouse monoclonal antibody against PANK2 yields a band at the expected 249 molecular weight (48 kDa), which disappears in the PANK2 CRISPR KO clones in HAP1, 
250 HeLa and LN319 cells (Figure 1A, E and F). However, we were unable to validate the

251 loss of the bands of PANK1 and PANK2 proteins in the CRISPR KO clones by

252 ProteinTech antibody (data not shown). We also tested multiple commercially available

253 antibodies against PANK3 using PANK3 knockout CRISPR cell lines as negative controls.

254 However, none of these antibodies gave the correct size band, and did not show loss of

255 the band in CRISPR PANK3 KO cell lines. This led us to invest in generating our PANK3 256 antibody.

\section{Production and Purification of His-tagged recombinant PANK3 protein.}

PANK3 is a $41 \mathrm{kD}$ cytosolic protein which is ubiquitously expressed in all cell types.

PANK3 protein is highly homologous between humans and rodents (homology with mice-

99.1\%, homology with rabbit-100\%) (Supplemental Figure S1), which necessitated the

maximal antigenic as well as conformational epitope recognition in mice. The pET28a plasmid encoding six Histidine residues fused to 364 amino acid residues of the human PANK3 protein (residues pro12 to Asn368) was used as a vector for recombinant PANK3 production (Figure 2A-C). pET28a plasmid was transformed into E. coli and His-PANK3 synthesis was induced by the addition of IPTG into the growth medium. The clarified

267 lysates from the E. coli cells were purified using a Ni-NTA column, and the eluted fractions were assessed for the presence of the recombinant PANK3 protein by Coomassie

269 staining of the SDS-PAGE (Figure 2A, C and D). An unidentifiable band was observed around $75 \mathrm{kDa}$, so the Ni-NTA column elutes were further subjected to a size exclusion

271 gel chromatography to obtain purified recombinant PANK3 protein (Figure 2E). 
To generate human PANK3 specific antibody, we immunized three mice-two NZB

274 mice and a BALB/c mouse, with recombinant PANK3 protein (Figure 3A). Due to a 99.1\%

275 sequence homology in PANK3 protein between human and mouse (Supplemental

276 Figure S1), we included the auto-immune NZB mouse model along with the wild type

277 BALB/c mouse for immunization. The mice were administered 5 doses of PANK3 antigen

278 mixed 1:1 with IFA every 2 days for the first 15 days, and then the final 3 booster doses

279 were administered every week (Figure 3A) The serum samples collected from the mice

280 were subjected to ELISA assay for quantification of serum PANK3 antibody titer. Due to

281

282

283

284

285

286

287

288

289

290

291

292

293 294 purification. As evidenced in Figure 4C, all four clones were able to detect the 295

high PANK3 antibody titer in NZB mouse \#2, we generated hybridomas using the plasma cells isolated from this mouse (Figure 3B-C). We fused the splenocytes isolated from the immunized mouse with the murine myeloma cells Sp20 and selected the positive hybridoma clones in the HAT medium (Figure 3C).

To determine the activity and efficacy of each hybridoma clone, we performed ELISA assay using the media supernatant from the hybridoma clones (Figure 4A-B). We further tested the efficacy and specificity of each clone-derived supernatant to detect endogenous PANK3 protein in HeLa cell lysates. Consistent with the ELISA results, the supernatants from most clones were able to recognize the recombinant antibody by immunoblotting assay (Figure 4C). In whole cell lysates, some of the clones were able to detect a band approximately at the expected molecular weight ( $41 \mathrm{kDa})$, however, none of the clones yielded clean and specific band for the endogenous PANK3 protein. We chose four clones that optimally recognized the endogenous PANK3 protein for further recombinant PANK3 protein, but the sub-optimal specificity for endogenous PANK3 and 
296 the presence of many non-specific bands posed significant challenges in further

297 application of the antibody. To mitigate these issues, we ventured on optimizing the 298 antibody and the immunoblotting assay conditions.

\section{Method-of-use optimization of MDA-299-62A anti-human PANK3 mouse mAb}

PANK3 is a cytosolic protein in eukaryotes (Figure 5A). To improve the specificity of

301 the antibody in immunoblotting assay, we performed subcellular fractionation to separate 302 cytosolic protein including PANK3 from the total cell lysate. As shown in Figure 5A-B, we 303 found that separating the cytosolic proteins by sub-cellular fractionation significantly reduced the non-specific signals and allowed reliable detection of endogenous PANK3 305 proteins in a broad range of cancer cell lines. Out of the four hybridoma clone 306 supernatants that we purified, clone MDA-299-62A appeared to be the most effective in 307 detecting endogenous PANK3 protein, with the most minimal signal to noise ratio. 308 Therefore, we used this clone for further optimization. To conclusively validate that the antibody was indeed recognizing the endogenous PANK3 protein, we employed the CRISPR Cas9 technology to knock out PANK3 protein in two different cancer cell lines-

311 HAP1 and HeLa (Figure 5B and C). We successfully obtained multiple single cell PANK3

312 knockout clones from these cell lines and validated that the antibody detected the correct

313 band (Figure 5B and $\mathbf{C}$ ). The CRISPR experiment also evidenced that PANK3 is a 314 dispensable gene in most cancer cells, which is consistent with large-scale CRISPR and 315 RNAi screen (DepMap) as well as mouse germline KO studies $(2,25)$. We also performed 316 knockdown studies using doxycycline inducible system and found that MDA-299-62A can

317 reliably detect shRNA mediated knockdown of PANK3 in cancer cells. 
While subcellular fractionation could eliminate the non-specific signals on the immunoblot, it can be a cumbersome experiment to perform for routine use of PANK3 antibody for immunoblotting. Intending to reduce the non-specific signals without the need

321 for subcellular fractionation, we modified the immunoblot assay protocol and employed a 322 longer blocking duration of 6-8 hours, compared to the conventional 1 hour at RT on

323 whole cell lysates (Figure $\mathbf{5 E}$ and $\mathbf{F}$ ). We found that the duration of blocking could 324 significantly dampen the non-specific signals and improved the signal to noise ratio in 325 both cytosolic protein fractions as well as whole cell lysates (Figure 5E and F).

\section{Discussion:}

CoA is an indispensable cofactor for a myriad of biochemical reactions in the cells. called PKAN in humans. Multiple therapeutic approaches are currently being explored in preclinical and clinical studies to restore CoA levels to combat CoA deficiency in these pathologies $(14,15)$. PANK proteins control the first and the rate-limiting step in the denovo coenzyme A biosynthesis. Owing to the essentiality of CoA at the cellular as well as organismal level, PANK and other CoA pathways proteins have also garnered interest as

334 attractive therapeutic targets against parasites such as Plasmodium falciparum and Toxoplasma gondii (26). The importance of CoA in microbes and the distinctions in CoA biosynthesis pathway proteins between microbes and mammals have also propelled 337 investigative interest on CoA biosynthesis as anti-bacterial targets $(27,28)$. Our drugtarget discovery investigations of PANK proteins also relies on the essentiality of PANK

339 proteins for CoA biosynthesis and aims to exploit the redundancies in the PANK proteins 
PTEN tumor suppressor locus, whose homozygous deletion is associated with poor

342 prognosis, high malignancy and resistance to both conventional chemotherapy and

343 precision oncology drugs (18-22). Despite significant investigative interest in

344 understanding the roles of PANK proteins in normal and pathological conditions, the

345 paucity of antibodies to detect endogenous PANK proteins has been a detriment to the

346 advancement of these studies.

In this study, we report the validation of commercially available PANK1 and PANK2

348 antibodies and demonstrate detection of band specificity using PANK1 and PANK2 349 CRISPR KO cells. Since we were unable to obtain any reliable PANK3 antibody against

endogenous human PANK3 protein, we generated and validated a human PANK3

351 specific mouse monoclonal antibody. We show that MDA-299-62A can reliably detect

352 recombinant PANK3 protein and endogenous PANK3 protein in ELISA and

353 immunoblotting assays. We confirmed the band specificity of our antibody by using a

354 PANK3 CRISPR knockout cell line, Our results show that the antibody MDA-299-62A can

355 be routinely used for immunoblotting assays, but with the caveat of multiple other 356 unspecific bands along with the PANK3 protein in the cell lysate (though this is also the 357 case with commercial anti-PANK2, mouse mAb (Origene \#TA501321, Figure 1E-F). The 358 use of full-length protein as an antigen possibly explains the detection of many unspecific 359 bands by the antibody. PANK3 is extremely conserved between humans and rodents 360 (rabbit 100\%). So, the antibody was raised in mice, which shares a $99.1 \%$ homology to 361 human PANK3 protein. We used the full-length protein in an autoimmune mouse model, 362 to allow for maximum antigen recognition and antibody production. While developing the 363 antibody in organisms with significant sequence differences could alleviate this issue, 
364 however, our attempt of developing PANK3 polyclonal antibody in chickens did not yield 365 improved results (data not shown).

To alleviate the issue of unspecific bands recognition by MDA-299-62A, we

367 demonstrate that by performing subcellular fractionation, we can concentrate PANK3 in

368 the cytosol fraction, which can significantly minimize unspecific bands. Since performing

369 sub-cellular fractionation on a routine basis can be cumbersome, we demonstrate that by

370 extending the duration of blocking of the membranes, we can significantly minimize these

371 unspecific bands. These mitigation measures are only relevant for immunoblotting

372 assays, and further optimization of MDA-299-62A mAb will be required for

373 immunohistochemistry, immunofluorescence as well as fluorescence activate cell sorting

374 (FACS) experiments. However, the consistent and reliable results that we obtained with

375 MDA-299-62A from well controlled experiments shows that the antibody is robust enough

376 to study the genetic interactions of PANK isoform knockout for the purposes of validating

377 PANK as collateral lethality targets in cancer.

$378 \quad$ Funding

379 We appreciate all financial support received, briefly: This work was supported by the NIH 380 R21CA226301 (F.L.M.), NIH SPORE 2P50CA127001-11A1(F.L.M), Elsa U. Pardee 381 Foundation (F.L.M.), The Uncle Kory Foundation (F.L.M), The Larry Deaven Fellowship, 382 MD Anderson UT Health Graduate School of Biomedical Sciences (S.K) and CPRIT 383 Research Training Grant (RP170067) (S.K), and the Cancer Answers/Sylvian Rodriguez 384 Scholarship (S.K.). The UT-MDACC Monoclonal Antibodies Core facility, is supported in 385 part by CCSG grant P30 CA016672.

\section{Author's contributions}


387 SK and FLM conceived the study. PL performed recombinant protein expression and 388 purification. LV and LB performed animal immunization, hybridoma generation and 389 antibody purification. SK performed all in-vitro genetic and antibody validation 390 experiments. SK wrote the manuscripts with assistance from FLM. All authors approved 391 the final manuscript.

392 Conflict of Interest

393 The authors declare that they have no conflict of interest with the contents of this article.

394 Data Availability

395 All data relevant to the manuscripts are included.

Acknowledgements

We thank Dr. Ronald DePinho for his guidance and research support on this work. We also want to thank Sarah Joseph for her help in generation and purification of recombinant PANK3 protein. The graphical abstracts and figures were adapted from or created with

400 Biorender.com.

401 References:

1. Leonardi, R., Zhang, Y. M., Rock, C. O., and Jackowski, S. (2005) Coenzyme A: back in action. Prog Lipid Res 44, 125-153

2. Dansie, L. E., Reeves, S., Miller, K., Zano, S. P., Frank, M., Pate, C., Wang, J., and Jackowski, S. (2014) Physiological roles of the pantothenate kinases. Biochem Soc Trans 42, 1033-1036

3. Gout, I. (2018) Coenzyme A, protein CoAlation and redox regulation in mammalian cells. Biochem Soc Trans 46, 721-728

4. Theodoulou, F. L., Sibon, O. C., Jackowski, S., and Gout, I. (2014) Coenzyme A and its derivatives: renaissance of a textbook classic. Biochem Soc Trans 42, 1025-1032

5. Jackowski, S., and Rock, C. O. (1981) Regulation of coenzyme A biosynthesis. J Bacteriol 148, 926932

6. Alfonso-Pecchio, A., Garcia, M., Leonardi, R., and Jackowski, S. (2012) Compartmentalization of mammalian pantothenate kinases. PLoS One 7, e49509

7. Vallari, D. S., Jackowski, S., and Rock, C. O. (1987) Regulation of pantothenate kinase by coenzyme A and its thioesters. J Biol Chem 262, 2468-2471 
8. Leonardi, R., Rehg, J. E., Rock, C. O., and Jackowski, S. (2010) Pantothenate kinase 1 is required to support the metabolic transition from the fed to the fasted state. PLoS One 5, e11107

9. Jackowski, S., and Leonardi, R. (2014) Deregulated coenzyme A, loss of metabolic flexibility and diabetes. Biochem Soc Trans 42, 1118-1122

10. Leonardi, R., Rock, C. O., and Jackowski, S. (2014) Pank1 deletion in leptin-deficient mice reduces hyperglycaemia and hyperinsulinaemia and modifies global metabolism without affecting insulin resistance. Diabetologia 57, 1466-1475

11. Corbin, D. R., Rehg, J. E., Shepherd, D. L., Stoilov, P., Percifield, R. J., Horner, L., Frase, S., Zhang, Y. M., Rock, C. O., Hollander, J. M., Jackowski, S., and Leonardi, R. (2017) Excess coenzyme A reduces skeletal muscle performance and strength in mice overexpressing human PANK2. Mol Genet Metab 120, 350-362

12. Subramanian, C., Yao, J., Frank, M. W., Rock, C. O., and Jackowski, S. (2020) A pantothenate kinasedeficient mouse model reveals a gene expression program associated with brain coenzyme a reduction. Biochim Biophys Acta Mol Basis Dis 1866, 165663

13. Sharma, L. K., Leonardi, R., Lin, W., Boyd, V. A., Goktug, A., Shelat, A. A., Chen, T., Jackowski, S., and Rock, C. O. (2015) A high-throughput screen reveals new small-molecule activators and inhibitors of pantothenate kinases. J Med Chem 58, 1563-1568

14. Zano, S. P., Pate, C., Frank, M., Rock, C. O., and Jackowski, S. (2015) Correction of a genetic deficiency in pantothenate kinase 1 using phosphopantothenate replacement therapy. Mol Genet Metab 116, 281-288

15. Sharma, L. K., Subramanian, C., Yun, M. K., Frank, M. W., White, S. W., Rock, C. O., Lee, R. E., and Jackowski, S. (2018) A therapeutic approach to pantothenate kinase associated neurodegeneration. Nat Commun 9, 4399

16. Hortnagel, K., Prokisch, H., and Meitinger, T. (2003) An isoform of hPANK2, deficient in pantothenate kinase-associated neurodegeneration, localizes to mitochondria. Hum Mol Genet 12, 321-327

17. Kotzbauer, P. T., Truax, A. C., Trojanowski, J. Q., and Lee, V. M. (2005) Altered neuronal mitochondrial coenzyme $A$ synthesis in neurodegeneration with brain iron accumulation caused by abnormal processing, stability, and catalytic activity of mutant pantothenate kinase 2 . J Neurosci 25, 689-698

18. Bucheit, A. D., Chen, G., Siroy, A., Tetzlaff, M., Broaddus, R., Milton, D., Fox, P., Bassett, R., Hwu, P., Gershenwald, J. E., Lazar, A. J., and Davies, M. A. (2014) Complete loss of PTEN protein expression correlates with shorter time to brain metastasis and survival in stage IIIB/C melanoma patients with BRAFV600 mutations. Clin Cancer Res 20, 5527-5536

19. Peng, W., Chen, J. Q., Liu, C., Malu, S., Creasy, C., Tetzlaff, M. T., Xu, C., McKenzie, J. A., Zhang, C., Liang, X., Williams, L. J., Deng, W., Chen, G., Mbofung, R., Lazar, A. J., Torres-Cabala, C. A., Cooper, Z. A., Chen, P. L., Tieu, T. N., Spranger, S., Yu, X., Bernatchez, C., Forget, M. A., Haymaker, C., Amaria, R., McQuade, J. L., Glitza, I. C., Cascone, T., Li, H. S., Kwong, L. N., Heffernan, T. P., Hu, J., Bassett, R. L., Jr., Bosenberg, M. W., Woodman, S. E., Overwijk, W. W., Lizee, G., Roszik, J., Gajewski, T. F., Wargo, J. A., Gershenwald, J. E., Radvanyi, L., Davies, M. A., and Hwu, P. (2016) Loss of PTEN Promotes Resistance to T Cell-Mediated Immunotherapy. Cancer Discov 6, 202-216

20. Catalanotti, F., Cheng, D. T., Shoushtari, A. N., Johnson, D. B., Panageas, K. S., Momtaz, P., Higham, C., Won, H. H., Harding, J. J., Merghoub, T., Rosen, N., Sosman, J. A., Berger, M. F., Chapman, P. B., and Solit, D. B. (2017) PTEN Loss-of-Function Alterations Are Associated With Intrinsic Resistance to BRAF Inhibitors in Metastatic Melanoma. JCO Precis Oncol 1

21. Muller, F. L., Aquilanti, E. A., and DePinho, R. A. (2015) Collateral Lethality: A new therapeutic strategy in oncology. Trends Cancer 1, 161-173 
22. Muller, F. L., Colla, S., Aquilanti, E., Manzo, V. E., Genovese, G., Lee, J., Eisenson, D., Narurkar, R., Deng, P., Nezi, L., Lee, M. A., Hu, B., Hu, J., Sahin, E., Ong, D., Fletcher-Sananikone, E., Ho, D., Kwong, L., Brennan, C., Wang, Y. A., Chin, L., and DePinho, R. A. (2012) Passenger deletions generate therapeutic vulnerabilities in cancer. Nature 488, 337-342

23. Muhsin, A., Rangel, R., Vien, L., and Bover, L. (2022) Monoclonal Antibodies Generation: Updates and Protocols on Hybridoma Technology. Methods Mol Biol 2435, 73-93

24. Wutz, A. (2014) Haploid animal cells. Development 141, 1423-1426

25. Garcia, M., Leonardi, R., Zhang, Y. M., Rehg, J. E., and Jackowski, S. (2012) Germline deletion of pantothenate kinases 1 and 2 reveals the key roles for CoA in postnatal metabolism. PLoS One 7, e40871

26. Schalkwijk, J., Allman, E. L., Jansen, P. A. M., de Vries, L. E., Verhoef, J. M. J., Jackowski, S., Botman, P. N. M., Beuckens-Schortinghuis, C. A., Koolen, K. M. J., Bolscher, J. M., Vos, M. W., Miller, K., Reeves, S. A., Pett, H., Trevitt, G., Wittlin, S., Scheurer, C., Sax, S., Fischli, C., Angulo-Barturen, I., Jimenez-Diaz, M. B., Josling, G., Kooij, T. W. A., Bonnert, R., Campo, B., Blaauw, R. H., Rutjes, F., Sauerwein, R. W., Llinas, M., Hermkens, P. H. H., and Dechering, K. J. (2019) Antimalarial pantothenamide metabolites target acetyl-coenzyme A biosynthesis in Plasmodium falciparum. Sci Transl Med 11

27. Gerdes, S. Y., Scholle, M. D., D'Souza, M., Bernal, A., Baev, M. V., Farrell, M., Kurnasov, O. V., Daugherty, M. D., Mseeh, F., Polanuyer, B. M., Campbell, J. W., Anantha, S., Shatalin, K. Y., Chowdhury, S. A., Fonstein, M. Y., and Osterman, A. L. (2002) From genetic footprinting to antimicrobial drug targets: examples in cofactor biosynthetic pathways. J Bacteriol 184, 45554572

28. Butman, H. S., Kotze, T. J., Dowd, C. S., and Strauss, E. (2020) Vitamin in the Crosshairs: Targeting Pantothenate and Coenzyme A Biosynthesis for New Antituberculosis Agents. Front Cell Infect Microbiol 10, 605662 
PANK3 Antibody generation and validation 


\section{Figure 1}
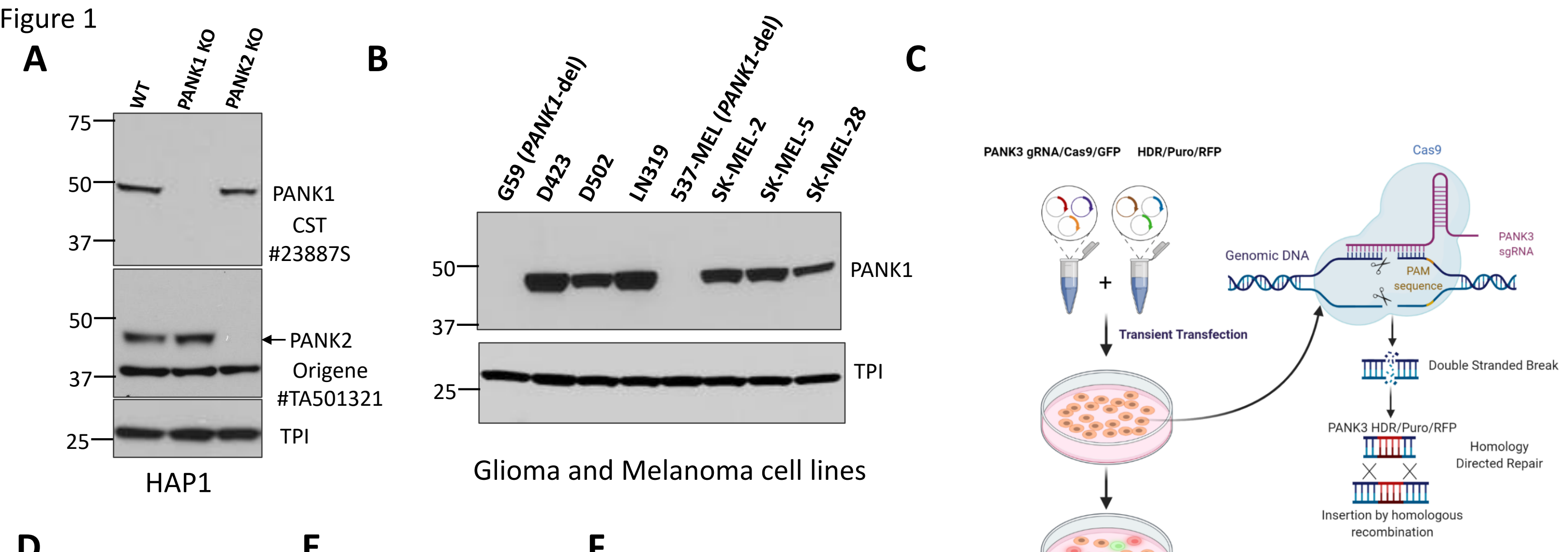

D
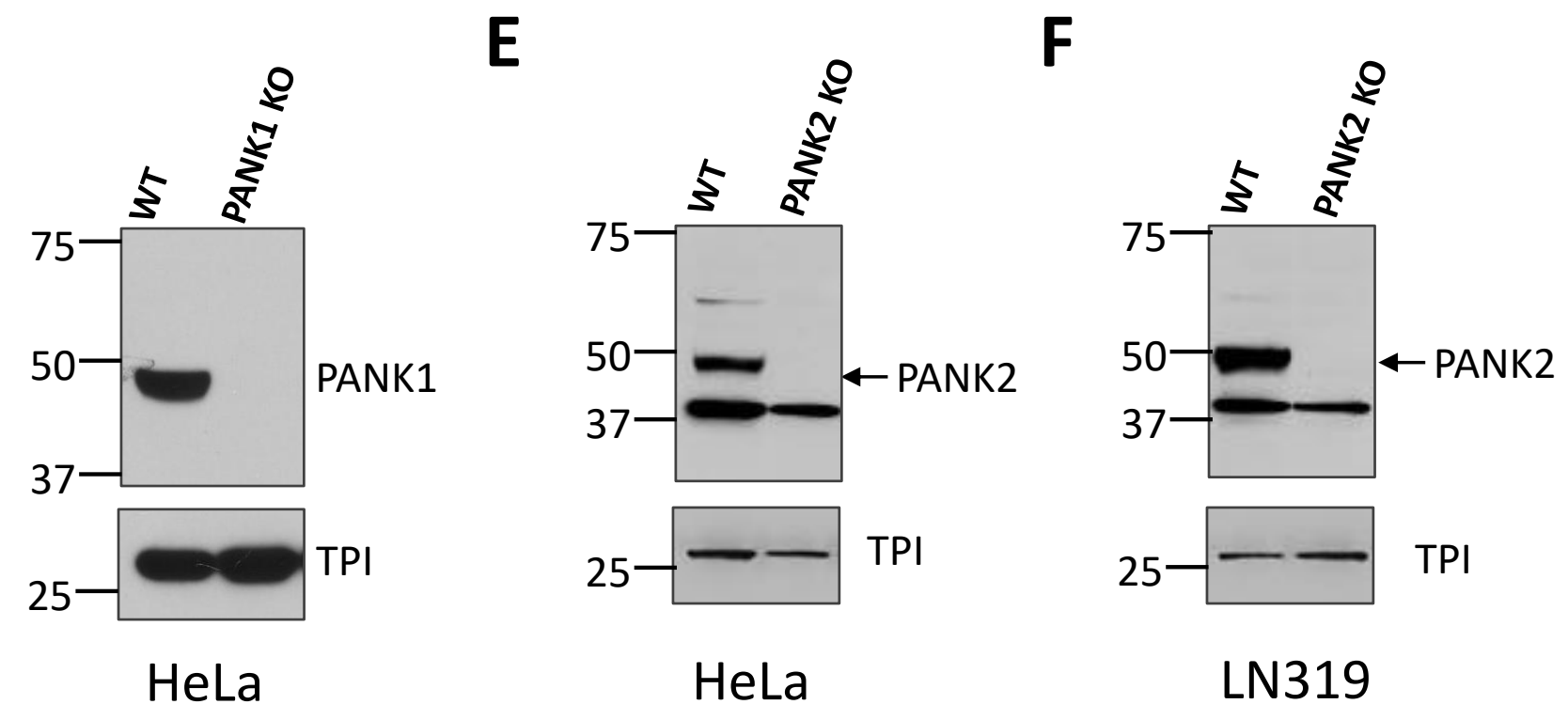

$48 \mathrm{~h}$ post transtection (1) Puromycin FACS single cell sorting

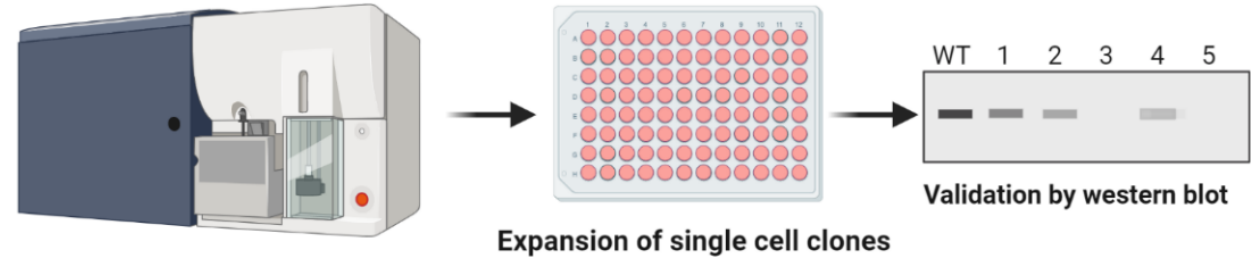
Expansion of single cell clones 


\section{Figure 1: Validation of commercially available antibodies for PANK1 and PANK2}

A. Commercially obtained and genomically-validated CRISPR knockout (KO) HAP1 cell lines were used as negative controls to verify specificity of PANK antibodies. Western blot confirming the loss of signal of PANK1 and loss of PANK2 in HAP1 PANK1 and PANK2 CRISPR KO clones purchased from Horizon Discovery. Rabbit anti-PANK1 mAb from CST (\#23887S) correctly identifies a single band at $50 \mathrm{kDa}$ (expected molecular weight of PANK1 protein) in WT HAP1 cells, and the band is absent in the PANK1 KO CRISPR clone. Origene mouse anti-PANK2 (mAb\#TA501321) identifies endogenous PANK2 protein in wild type HAP1 cells at the expected size of $48 \mathrm{kD}$, and the band disappears in PANK2 CRISPR KO clone. A non-specific band at $37 \mathrm{kD}$ is evident but is sufficiently distinct not to confound interpretation. Triose Phosphate Isomerase (TPI) is used as a loading control. B. Validation of CST PANK1 antibody using the PANK1 deleted and PANK1 intact glioma and melanoma lines. G59 (glioma) and $537 \mathrm{MEL}$ (melanoma) are PANK1 homozygous deleted cancer cell lines. $\mathbf{C}$. Schematic showing the Santa Cruz two-plasmid (gRNA/Cas9/GFP and HDR/Puromycin/RFP) system mediated CRISPR KO. D-F. Independent in-house generation of PANK1 and PANK2 CRISPR KO in cancer cells, and validation by western blot. 
Figure 2

Transformation

A

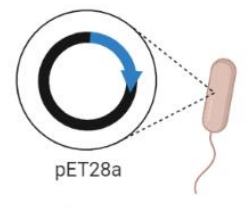

$\downarrow$ Selection

Protein production

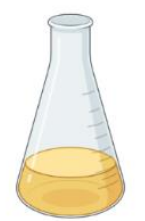

$\downarrow$ Cell lysis
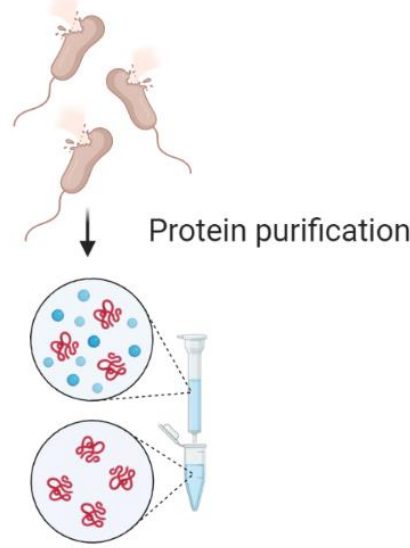

Analysis of purification

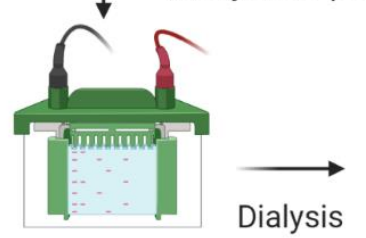

\section{PANK3 coding sequence}

1 MKIKDAKKPS FPWFGMDIGG TLVKLSYFEP IDITAEEEQE EVESLKSIRK YLTSNVAYGS

61 TGIRDVHLEL KDLTLFGRRG NLHFIRFPTQ DLPTFIQMGR DKNFSTLQTV LCATGGGAYK 121 FEKDFRTIGN LHLHKLDELD CLVKGLLYID SVSFNGQAEC YYFANASEPE RCQKMPFNLD 181 DPYPLLVVNI GSGVSILAVH SKDNYKRVTG TSLGGGTFLG LCSLLTGCES FEEALEMASK

241 GDSTQADKLV RDIYGGDYER FGLPGWAVAS SFGNMIYKEK RESVSKEDLA RATLVTITNN 301 IGSVARMCAV NEKINRVVFV GNFLRVNTLS MRLLAYALDY WSKGQLKALF LEHEGYFGAV 361 GALLGLPNFS

\section{Fractions from Ni-NTA column}

D

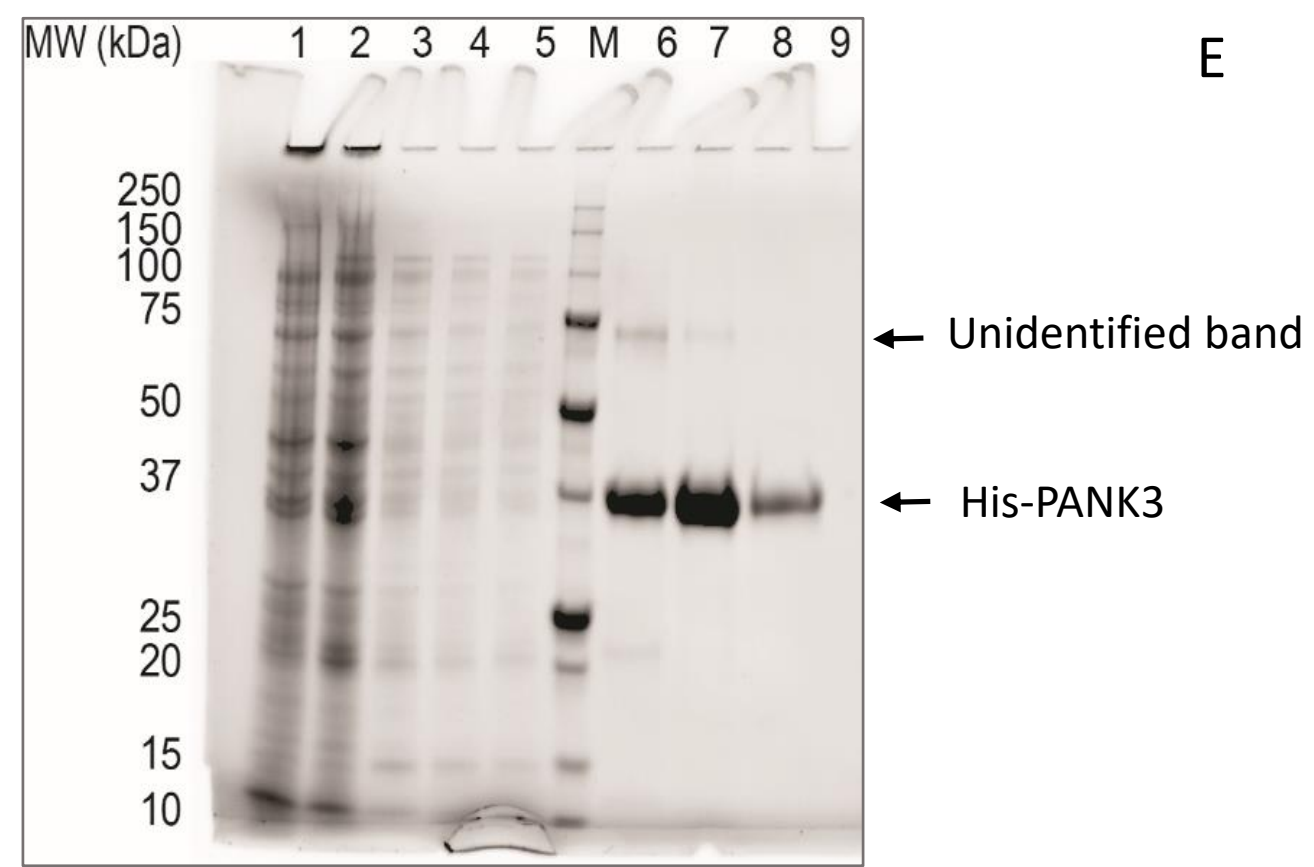

C

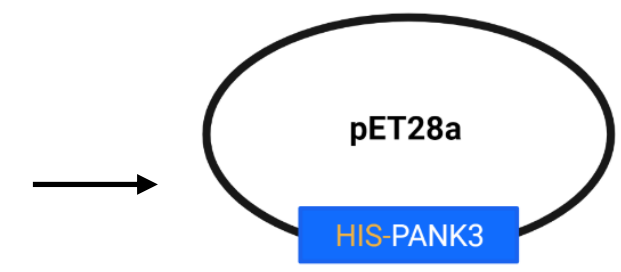

Pooled after gel filtration chromatography

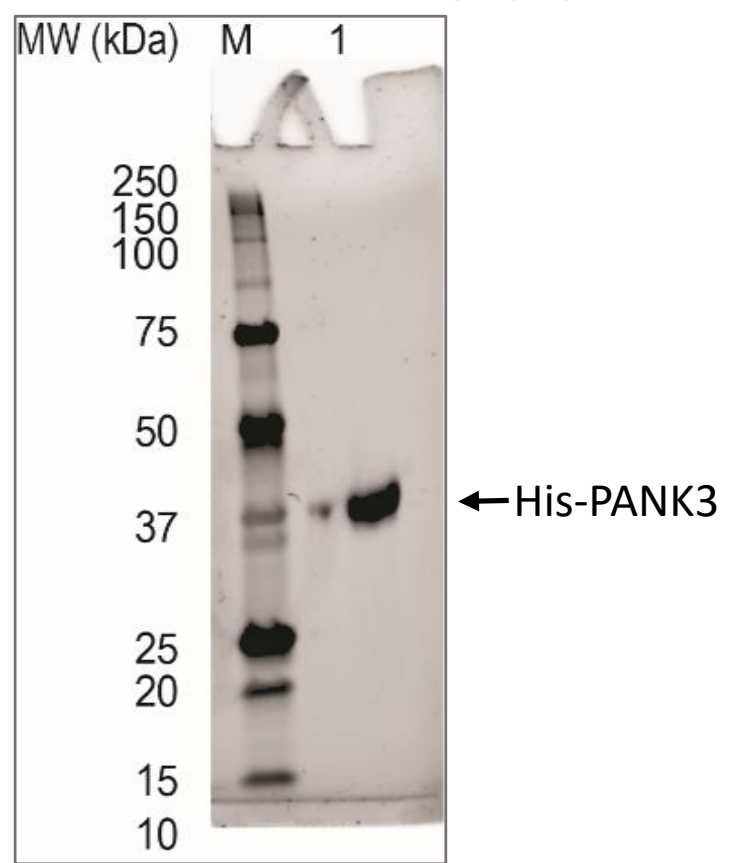




\section{Figure 2: Generation and purification of recombinant PANK3 protein}

A-C. Schematic showing the recombinant PANK3 production and purification. E. coli BL21 (DE3) and E. coli Rosetta2 (DE3) strains were transformed with pET28a plasmid vector encoding $\mathrm{N}$ terminal His tagged fusion PANK3 protein (residues pro12 to Asn368- highlighted in orange). The E. coli cells were grown in Terrific Broth media at $37^{\circ} \mathrm{C}$ overnight and then the temperature of the culture was reduced to $18^{\circ} \mathrm{C}$ prior to induction of the recombinant protein expression by the addition of $1 \mathrm{mM}$ IPTG. Cells were harvested and lysed, and the soluble supernatant was applied in a pre-equilibrated Ni-NTA column, to allow the histidine tag to bind to the column. After washing the column, the bound proteins were eluted in 10 different fractions. D-E. SDS PAGE gel was stained with Coomassie Blue to assess the presence of His-PANK3 in the fractions. PANK3 protein purified from the Ni-NTA column was further subjected to gel filtration chromatography. Purified PANK3 protein eluted through gel filtration was validated by SDS-PAGE gel and coomassie staining. 
A

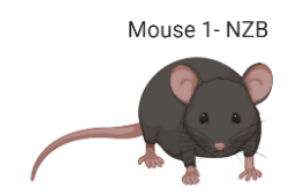

Mouse 2-NZB

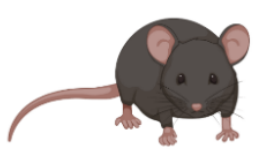

Mouse 3-BALB/C
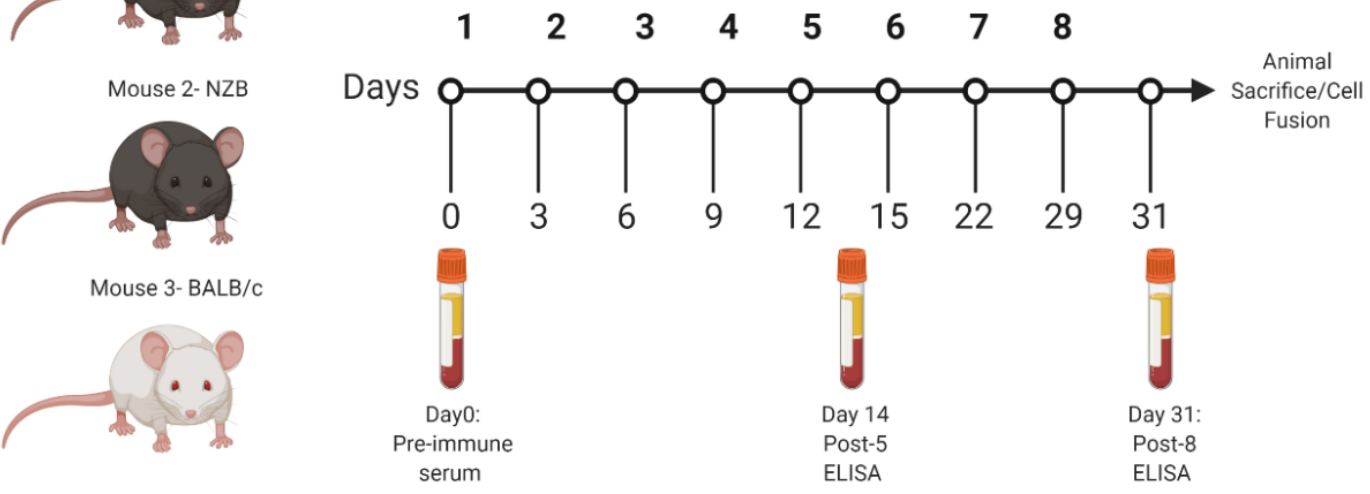

C

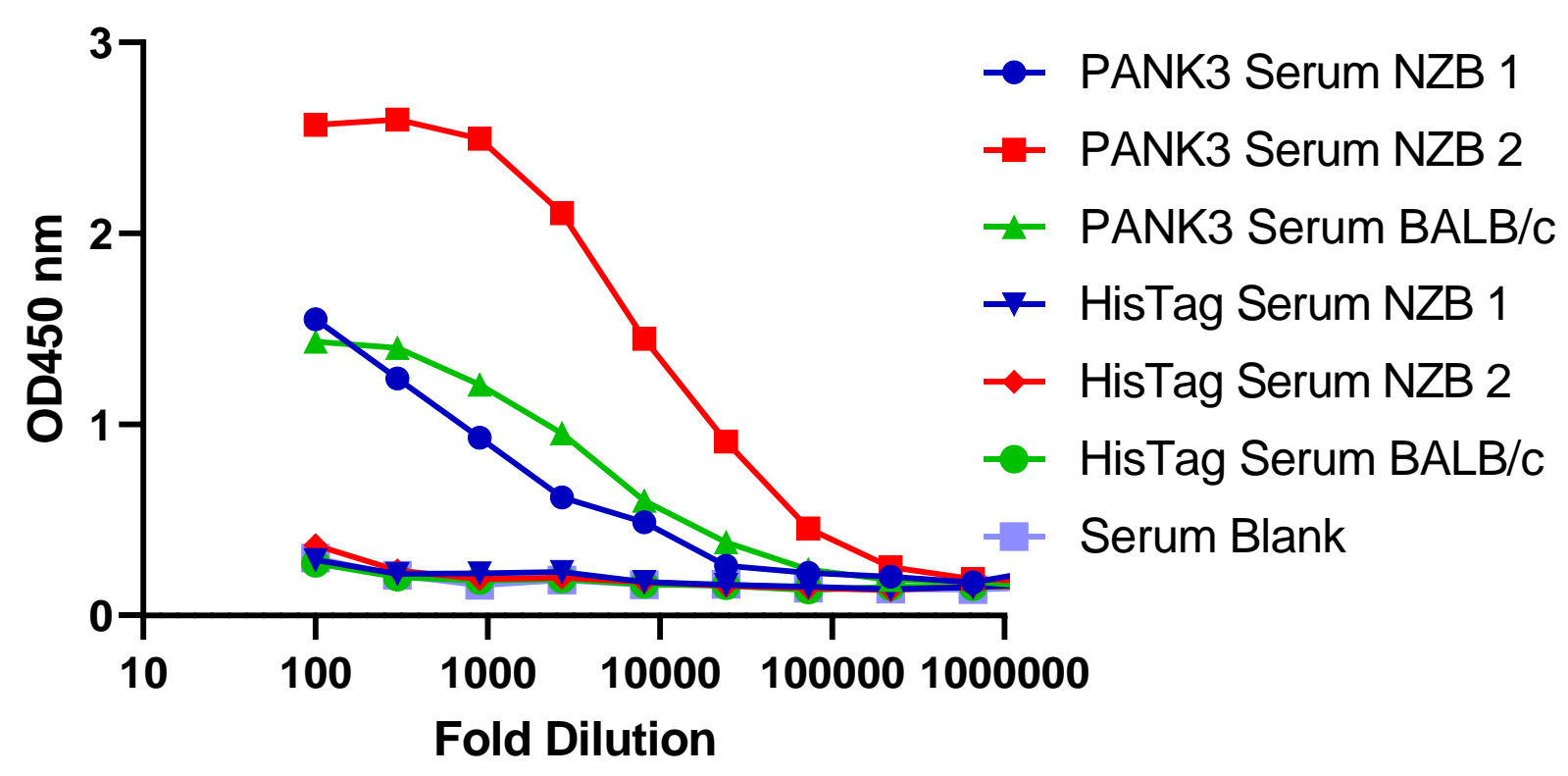

B Schematic of antibody generation

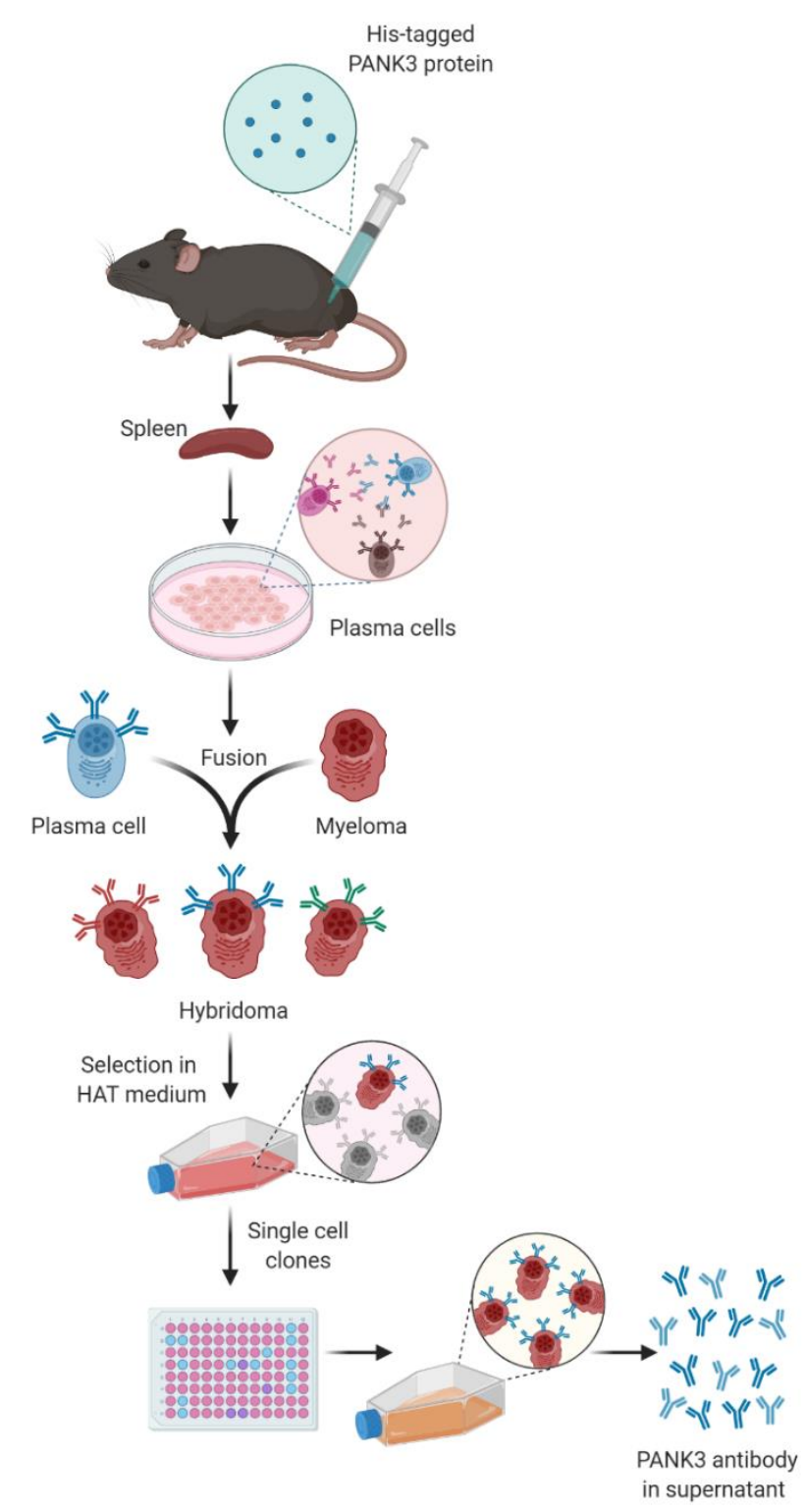




\section{Figure 3: Generation of PANK3 antibody and detection of serum antibody levels by ELISA}

A. Schematic showing the schedule of PANK3 protein immunization and blood collection for ELISA. PANK3 was administered in the foot pad of three mice, two NZB mice and a BALB/c mouse every two days for the first five doses and then weekly for three booster doses. Blood was collected on day 0,14 and 31 to determine antibody concentration in the serum by ELISA. B. Schematic depicting the general workflow for the generation of PANK3 antibody. Briefly, recombinant 20 ul human PANK3 protein and IFA mix was injected into the foot pad of 3 mice (two NZB and one BALB/c). Following eight injections, mice were euthanized and plasma cells from the spleen were isolated and were fused with murine myeloma cells, Sp20 to form fusion hybridoma cells. After selection in HAT medium, the antibody producing plasma cells were single cell cloned and media supernatant from each clone was subjected to ELISA screen. C. Serum titration of anti-PANK3 antibody collected before mice sacrifice on day 31 using ELISA assay. Serum levels of PANK3 antibody was higher in mouse no.2, which was used for subsequent hybridoma generation, single clone isolation and antibody production. Serum response to His tag is compared to blank. 
A

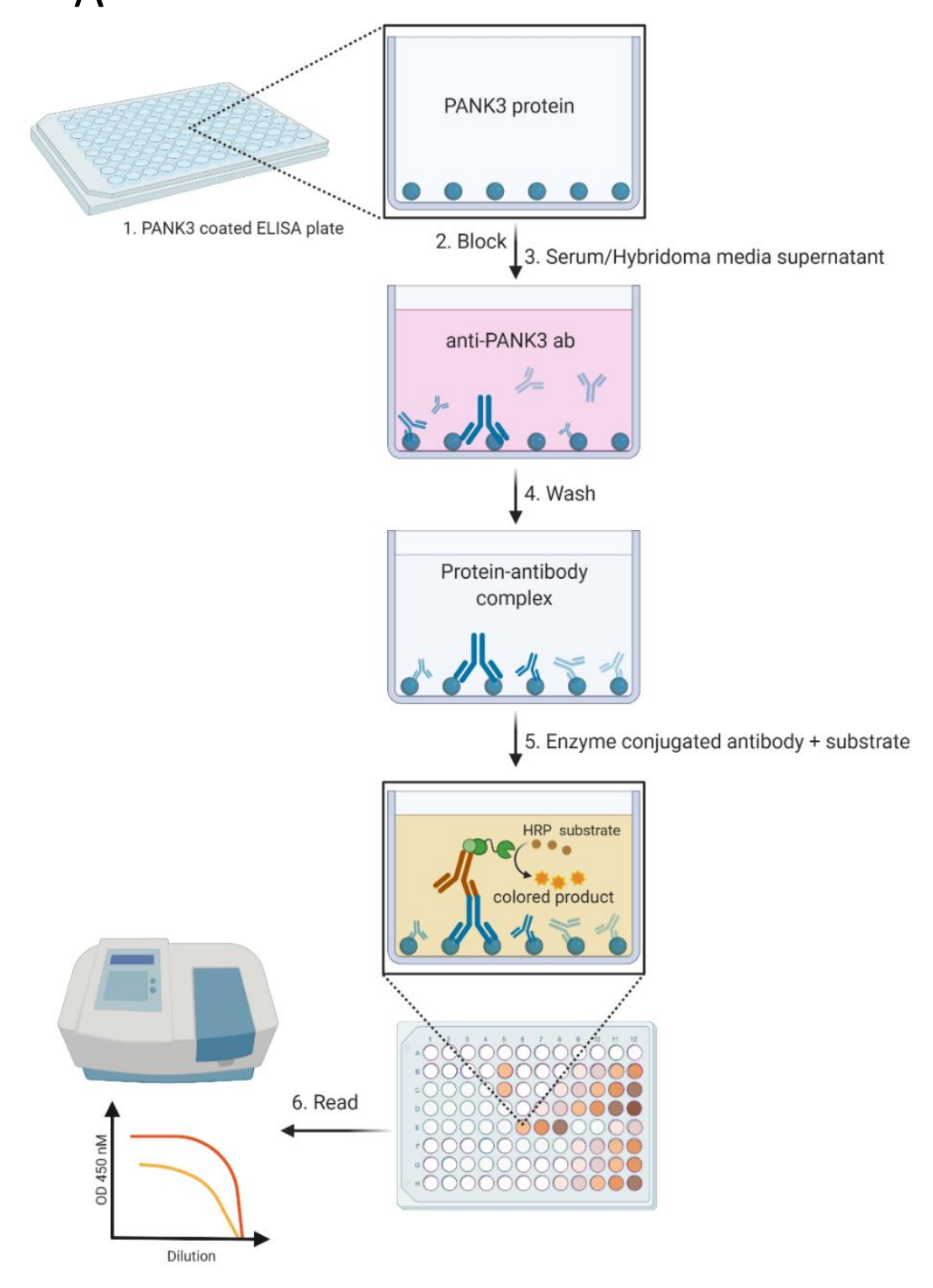

B

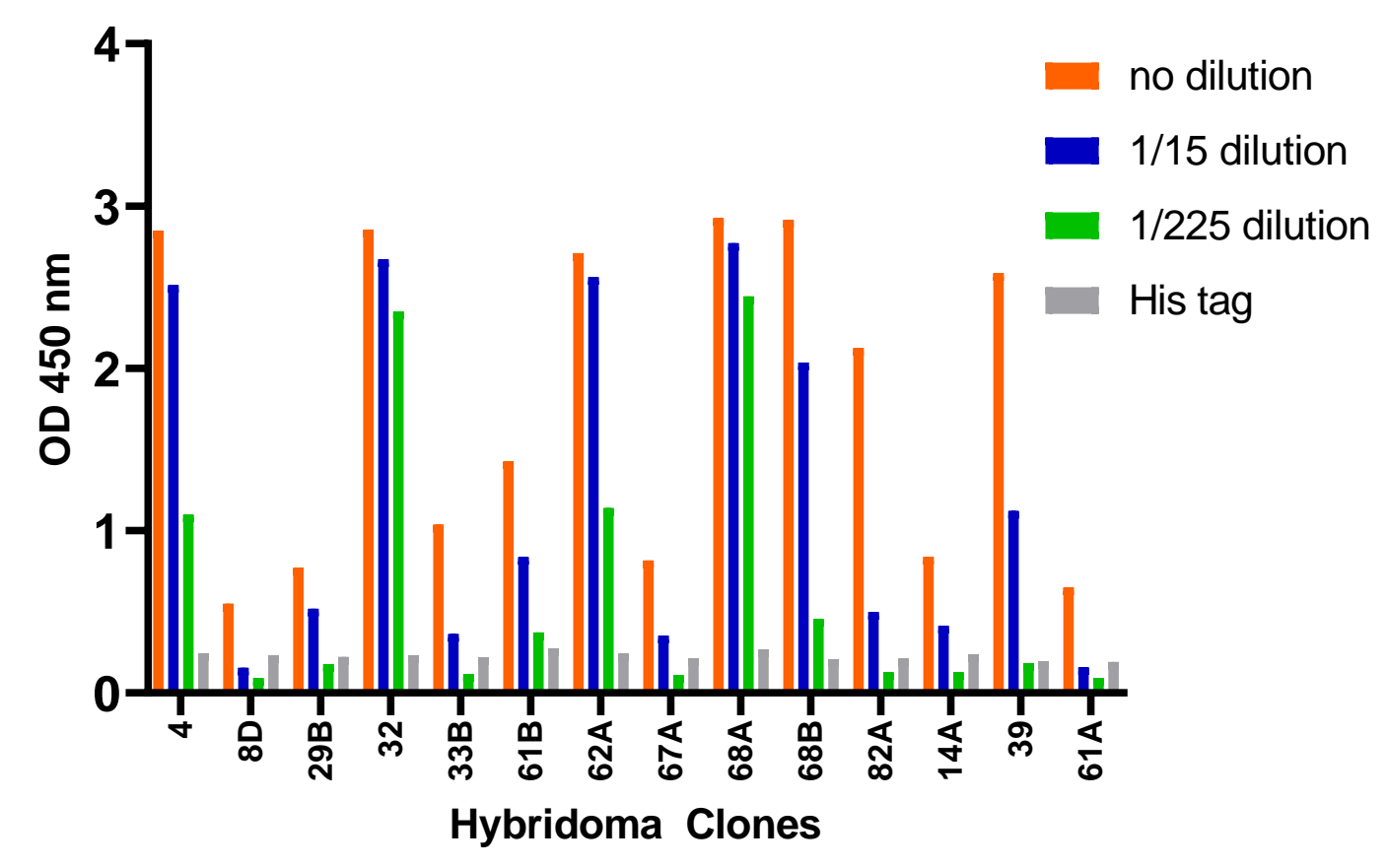

C

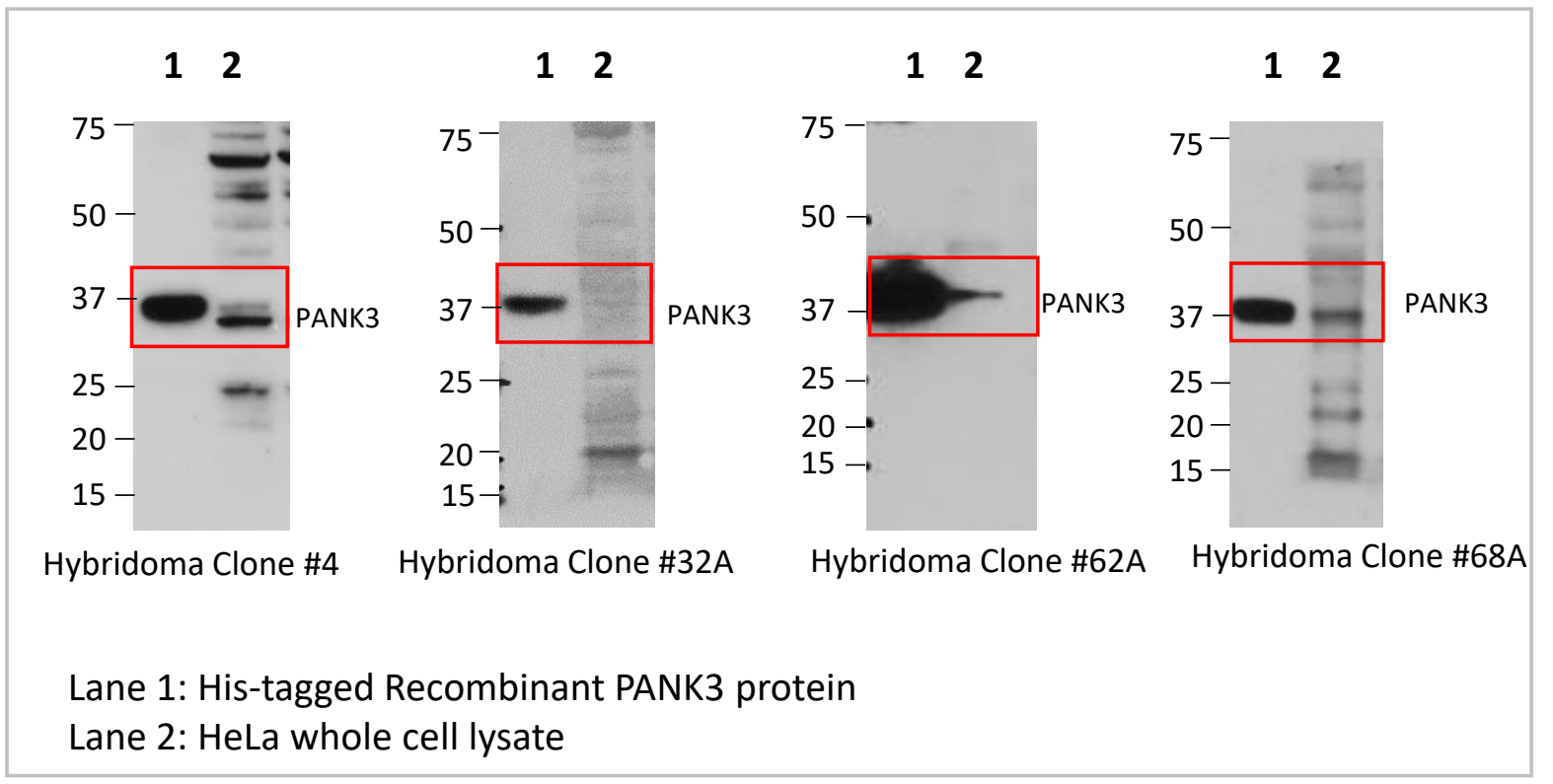




\section{Figure 4: Quantification of PANK3 antibody levels in media supernatant of hybridoma clones}

A. Schematic showing the detection of PANK3 protein by ELISA. Briefly, ELISA plates were coated with recombinant PANK3 protein and media supernatant (primary antibody) isolated from different hybridoma clones or the serum extracted from the immunized mice were added to the wells. An HRP linked secondary antibody was added to detect the PANK3 antibody bound to the immobilized PANK3 protein on the well. Addition of the substrate yielded a color change and the absorbance was quantified using a spectrophotometer. B. Absorbance values of medium supernatant (1:15 serial dilutions) isolated from different hybridoma clones. Only 14 clones out of 27 are shown in the graph. (See supplemental for all 27 clones). C. Immunoblot showing 4 antibody clones that were selected for further purification. The antibody clones are able to recognize recombinant protein, but the endogenous protein band in HeLa cell lysate is convoluted by overlapping non-specific bands. 


\section{Figure 5}
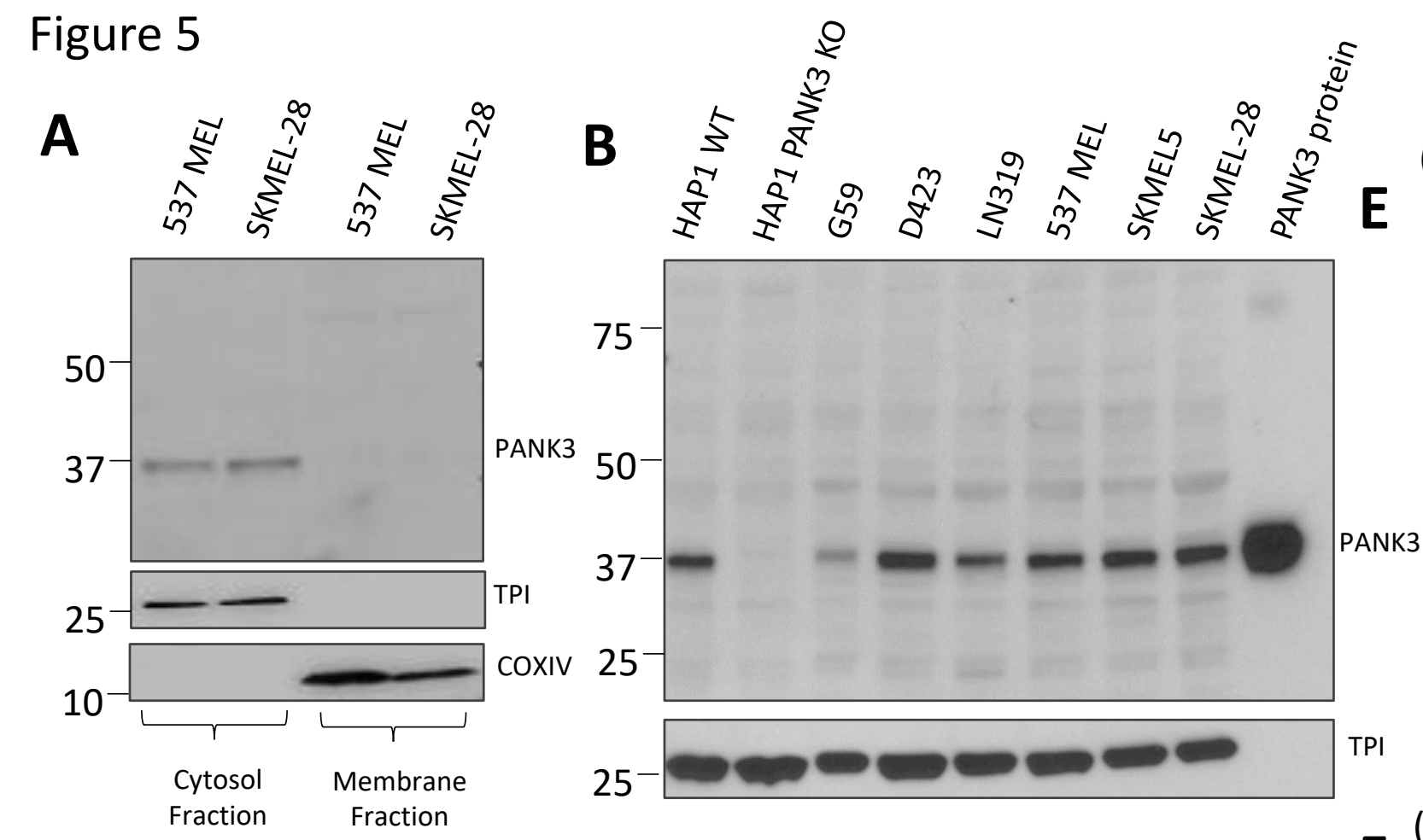

(Short exposure)

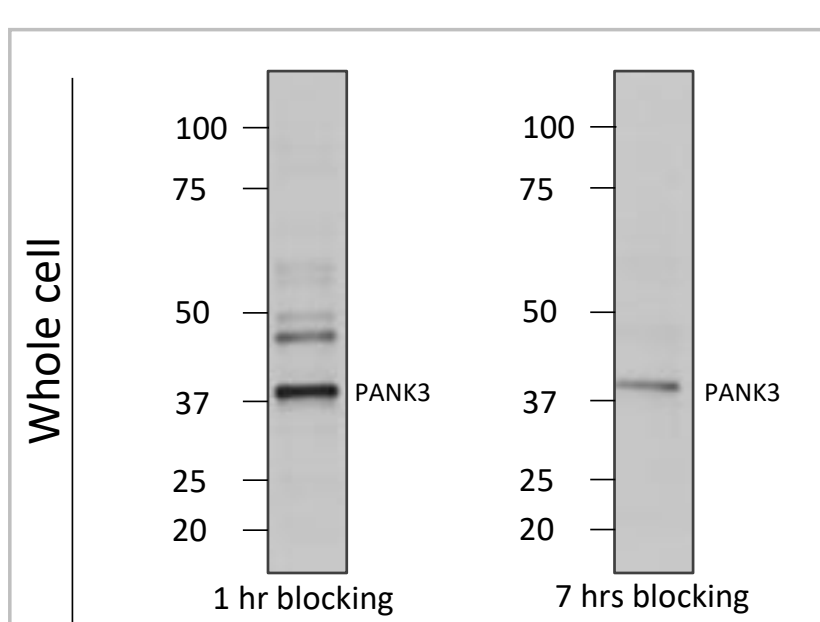

537 MEL cells
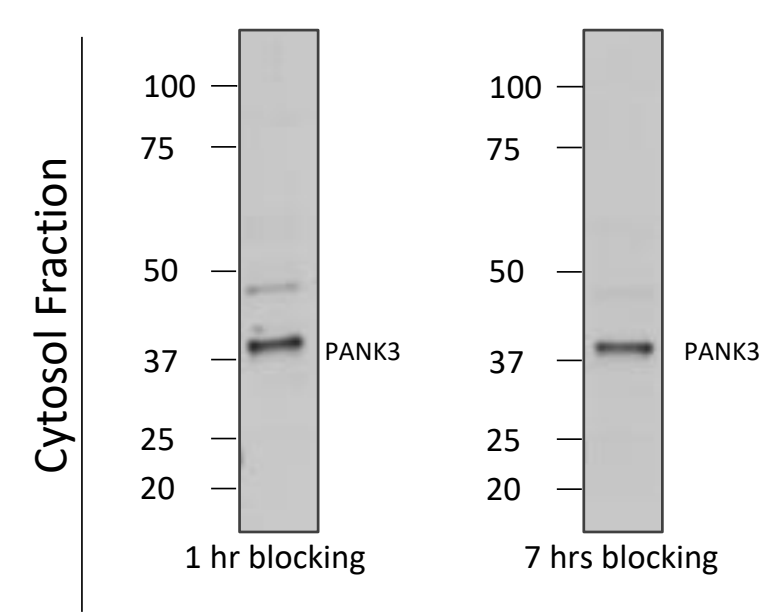

F (Long exposure)

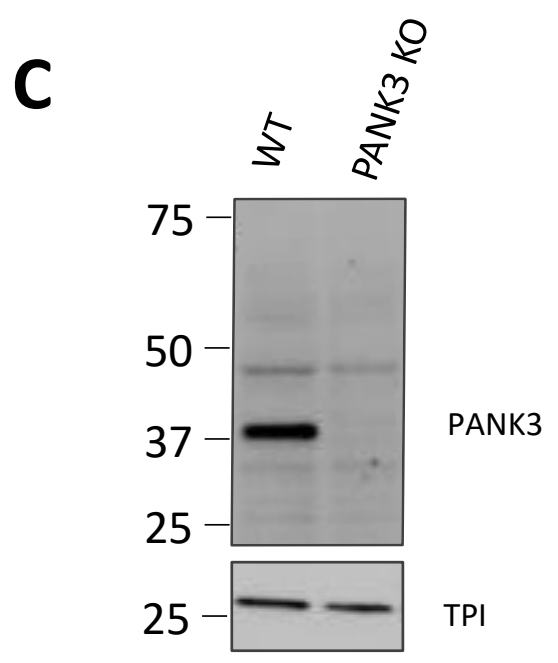

D
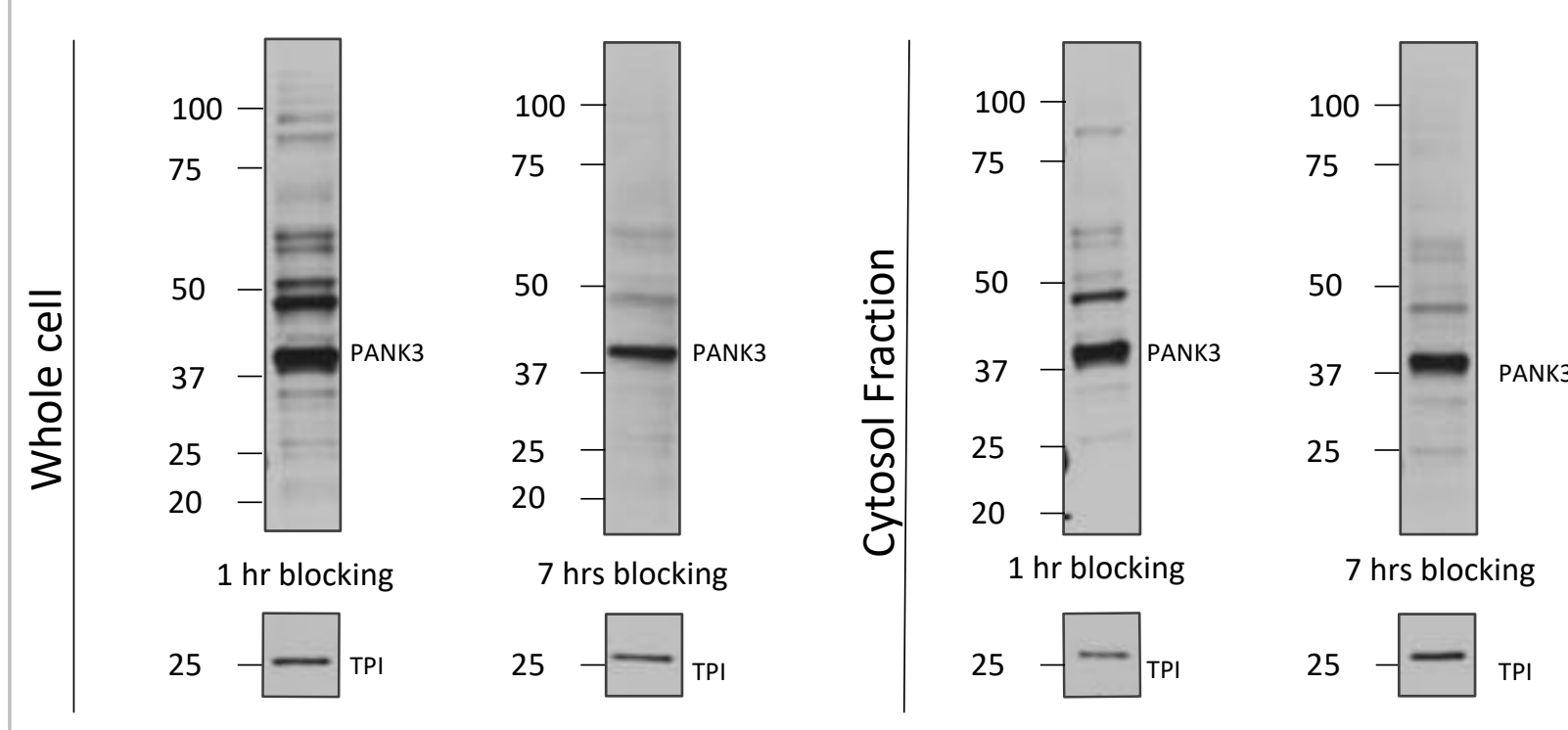


\section{Figure 5: Optimization of PANK3 specific detection by subcellular fractionation of cells and longer blocking of transferred membranes}

A. Immunoblots showing that PANK3 protein is found exclusively in the cytosolic fraction, confirming the cytosolic distribution of PANK3 protein in human cells. B. MDA-299-62A can detect the recombinant PANK3 protein (positive control) and endogenous PANK3 protein in cytosolic fractions of specified cancer cell lines. HAP1 PANK3 CRISPR KO(B) and HeLa PANK3 KO (C) cell lines were used as negative controls to verify correct band specificity. D. Knockdown of PANK3 protein by dox inducible shRNA, determined by immunoblot on cytosolic protein fractions using MDA-299-62A mouse monoclonal PANK3 antibody. E-F. The PANK3 band is detectable on whole cell lysates and the interference by non-specific bands is significantly reduced by increasing the duration of blocking to 7 hours instead of the typical 1 hour. Both whole cell lysates and cytosolic protein fractions are shown for reference, with shorter (15 secs) and longer exposure (5 minutes) of the $x$-ray film. 